\title{
CNS wide simulation of flow resistance and drug transport due to spinal microanatomy
}

\section{Abbreviated Title: Spinal Microanatomy Effect on Cerebrospinal Fluid Flow}




\section{Abstract}

Spinal microstructures are known to substantially affect cerebrospinal fluid patterns, yet their actual impact on flow resistance has not been quantified. Because the length scale of microanatomical aspects is below medical image resolution, their effect on flow is difficult to observe experimentally. Using a computational fluid mechanics approach, we were able to quantify the contribution of micro-anatomical aspects on cerebrospinal fluid (CSF) flow patterns and flow resistance within the entire central nervous system (CNS). Cranial and spinal CSF filled compartments were reconstructed from human imaging data; microscopic trabeculae below the image detection threshold were added artificially. Nerve roots and trabeculae were found to induce regions of microcirculation, whose location, size and vorticity along the spine were characterized. Our CFD simulations based on volumetric flow rates acquired with Cine Phase Contrast MRI in a normal human subject suggest a 2-2.5 fold increase in pressure drop mainly due to arachnoid trabeculae. The timing and phase lag of the CSF pressure and velocity waves along the spinal canal were also computed, and a complete spatio-temporal map encoding CSF volumetric flow rates and pressure was created.

Micro-anatomy induced fluid patterns were found responsible for the rapid caudo-cranial spread of an intrathecally administered drug. The speed of rostral drug dispersion is drastically accelerated through pulsatile flow around microanatomy induced vortices. Exploring massive parallelization on a supercomputer, the feasibility of computational drug transport studies was demonstrated. CNS-wide simulations of intrathecal drugs administration can become a practical tool for in silico design, interspecies scaling and optimization of experimental drug trials.

44 Keywords: Cerebrospinal Fluid Dynamics, Computational Fluid Dynamics, CNS microanatomy, MR image reconstruction, Drug dispersion, intrathecal drug delivery 


\section{Introduction}

Despite precise MR observations of cerebrospinal fluid (CSF) velocities ${ }^{18}$, brain motion ${ }^{46}$ and ventricular wall dilatations ${ }^{6}$, critical questions remain about CSF dynamics in the CNS: What is the exact nature of the force coupling between the pulsatile blood circulation and CSF

50 displacements? ${ }^{24,25}$ How is the pulsatile expansion and contraction of the cerebrovascular bed 51 transmitted to the CSF and the brain? The source of the volumetric dilations that actually induce 52 CSF motion remain uncertain, even though major cerebral arteries, the oscillatory displacement 53 by the parenchyma or choroid plexus expansion are likely involved. Moreover, the caudal 54 decrease in CSF flow amplitude along the spinal canal ${ }^{2,40}$ supports the notion of compliant leptomeningeal boundaries lining the fluid-filled spinal subarachnoid space (SAS). Hence, the observed CNS compliance could result from cyclic displacement of blood inside large spinal veins, or the expansion of CSF-filled spaces against elastically deformable epidural tissues. Nevertheless, the biomechanics of deformations of the entire spinal compartment remain unclear. diagnostics of pathological conditions. For example, the amplitude ratio between the aqueduct 61 and prepontine CSF flows were found elevated in hydrocephalus patients compared to normal subjects. ${ }^{46}$ Because in vivo MR measurements have limited image resolution and practical scan 63 durations restrict the number of observed regions of interest, many researchers have attempted to 64 address fundamental questions regarding CNS with computational fluid dynamics (CFD) 65 techniques dynamics especially under pathological conditions.

66 Early CFD models used small sections of the CNS with idealized geometries. ${ }^{7,16,17,19,22}$ Other 67 approaches used medical image reconstruction to represent subjects-specific anatomical spaces 68 to enhance the model fidelity. ${ }^{9,21,31,32,34,38,39}$ Haller et al. showed that spinal microanatomy 
induces complex mixing eddies. ${ }^{10}$ Stockman studied the impact of nerve roots and trabeculae on convective flow with Lattice Boltzman simulations in idealized cylindrical spine models. ${ }^{35,36}$ Gupta presented an analytical solution to oscillating fluid in a representative elliptical annulus representing a spinal segment directly inferior to the cisterna magna. ${ }^{8}$ Based on a simulated porous model of the cerebromeduallary cistern and spinal SAS, they conclude that subresolution microstructure density and radius can triple the pressure drop in CSF flow. ${ }^{8,9}$

Several groups investigated CSF flow patterns in Chiari patients relative to normal subjects. ${ }^{26,31,32}$ Yiallourou $^{45}$ et al. compared flow measurements in normal subjects and patients suffering from Chiari malformation with a CFD model of a cervical segment of the spine. During the review process of this paper, an updated report to the earlier CFD simulation paper of the same group including artificial nerve roots appeared. ${ }^{11}$ Yet none of these studies performed CFD simulations on the entire CNS and included microanatomical trabeculae in the spinal SAS.

The goal of this paper is to address several critical questions regarding the spatiotemporal coupling of the cranio-spinal CSF compartments by performing CFD simulation on an entire CNS model of a normal subject. This study tests the hypothesis that microanatomical structures generate significant vortex phenomena in spinal CSF flow which increase flow resistance, raise pressure drop, and induce complex mixing patterns responsible for the rapid biodispersion of moieties administered to the spinal SAS. To account for the influence of detailed spinal anatomical structures, nerve roots were reconstructed from medical images; arachnoid trabeculae below the imaging threshold were artificially incorporated to the subject-specific computational mesh. This paper constitutes a first step towards a complete in silico model of CNS dynamics with full coupling between CSF, blood and brain. To augment the limited scope of previous studies, it is critical to account for the entire CNS including the cranial and spinal SAS with their 
92 microscopic aspects. Modeling the complete CNS eliminates the burden of introducing uncertain

93 assumptions about internal boundary conditions. Therefore, a parametric study to quantify the

94 influence of microanatomical aspects on CSF dynamics and flow resistance and drug transport 95 was conducted. specific computational model of the cranial and spinal CSF-filled spaces including nerve roots was reconstructed from medical images, up to detail limited by resolution of the dataset using MIMICS image processing software (Appendix 1.1). CSF flow measurements were acquired from the 29 year old volunteer with CINE MRI at three planes in the spinal SAS (Appendix 1.2). ventricles were periodically displaced to account for pulsatile expansion and contraction of the cerebral vasculature. Explicit deformable boundaries were also applied at the thoracic and lumbar spine (Appendix 1.3). To assess the impact of microanatomical features below the imaging resolution, arachnoid trabeculae were artificially added to a $3 \mathrm{~cm}$ long cervical spine segment, visualized in Fig. 1 and discussed in Appendix 1.4. Fluid flow was solved with ANSYS

11014.0 Fluent on Blacklight at the Pittsburgh Supercomputing Center (Appendix 1.5). Fluid flow 111 parameters such as volumetric flow rate, stroke volume, Womersley, and Reynolds number are 112 calculated at each plane to characterize flow profiles and compare with MRI measurements 113 (Appendix 1.6). Upon close inspection of the original MR images and the reconstructed subject114 specific models the authors determined that the geometry was reasonably symmetrical along the 
115 mid-sagittal plane and utilized a symmetry boundary condition to reduce mesh size and 116 computation time.

117 Drug transport simulations. To assess the impact of nerve root induced CSF mixing 118 patterns, intrathecal (IT) drug infusion in the lumbar region was simulated in multiple scenarios;

119 more details on the IT drug delivery protocol can be found in prior work. ${ }^{14}$ The advection120 diffusion of bupivacaine in eq 1 was solved for a period of 12 hours post injection. Here, the 121 drug concentration is $C(x, t)$, and the diffusivity is $D=2.1 * 10^{-10} \mathrm{~m}^{2} \mathrm{~s}^{-1}$. The pulsating convective 122 CSF flow field, $u(x, t)$, was computed as described above. Drug metabolism and uptake were 123 considered negligible Species Transport

$$
\partial C \partial t+\nabla \cdot u x, t C=\nabla \cdot D \nabla C
$$
interest for a full cardiac cycle, in Fig. 2. Systole lasts about $25-35 \%$ of the cardiac cycle with a sharp forceful peak in the craniocaudal direction, this phase is color coded in red. Diastole covers

131 the remainder of the cardiac cycle with a slower, more even flow in the caudocranial direction, 132 color coded in blue. Regions of low velocities, $\|\mathrm{v}\|<0.1 \mathrm{~cm} \mathrm{~s}^{-1}$, are coded in green to highlight 133 temporary stagnation and transition of flow direction. Simulated peak plane-averaged velocities 134 for systole and diastole are presented in Table 1. The pressure wave depicted in Fig. 3 shows a short forceful peak during systole followed 136 by reversal during the slower diastolic phase. A fast traveling wave enters the spinal cord 137 cranially and can be traced caudally. The timing of the pressure in the spinal SAS follows the 
138 pulsatile dynamics of cerebro-arterial expansion. MR imaging has shown that during systolic

139 vessel expansion, fast craniocaudal CSF displacements can be observed in the aqueduct, the

140 cisterna magna and the cervical SAS, while during diastole there is flow reversal due to the

141 rostral recoil of the spinal CSF. ${ }^{15}$ Also, peak pulse amplitude becomes gradually smaller in the

142 caudal direction. In the cervical region, amplitudes are 223/99 Pa (sys/diast), reduce to 129/52 in

143 the thoracic, and drop to $45 / 18$ in the lumbar region. Pressure predictions for all axial planes are

144 listed in Table 1.

145 Volumetric flow profiles and stroke volume ratios are visualized in Fig. 3. Peak 146 volumetric flow rates and stroke volume decrease caudally along the spine. The simulated 147 volumetric flow rate lags behind the pressure wave across the entire spine with the least at CM, 148 phase angle $7.2^{\circ}$, and greatest at $\mathrm{S} 3$ with phase angle of $64.8^{\circ}$; phase angles for all regions are 149 reported in Table 1. Phase angle at each plane is calculated as the peak-to-peak difference 150 between pressure and velocity waves. CSF phase lag is not well characterized; Wagshul et al. 151 reported values at the $\mathrm{C} 2$ level, $+10.8 \pm 11.2^{\circ}$ (posterior) and $-0.5 \pm 10.8^{\circ}$ (anterior). Our $\mathrm{C} 4$ phase 152 lag is $10.8^{\circ}$, comparable to their reported range. ${ }^{42}$ Womersley numbers were calculated using 153 eq. 11 (Appendix 1.6) to characterize flow conditions due to a pulsatile pressure gradient.

154 Fig. 4 plots trajectories of volumetric flow rates for one cardiac cycle acquired in the 155 volunteer (thick lines). For comparison, simulation results are displayed as filled curves. Flow 156 profiles of our MRI data and CFD simulations depict caudal flow as red and cranial flow as blue.

157 At the $\mathrm{C} 4$ level, both measured and simulated profiles have a rapid increase in caudal flow, 158 which tapers off over $25-35 \%$ of the cardiac cycle. Cranial flow for MR data and predicted 159 values is gradual with lower peak amplitudes than systole. MRI stroke volume and predicted 160 values match well for all planes. The difference in the stroke volume between simulated 
161 magnitudes and MRI data is less than $0.004 \mathrm{~mL}$ for all planes, yielding a percent difference of $1620.9 \%$ at $\mathrm{C} 4,1.6 \%$ at $\mathrm{T} 6$, and $1.5 \%$ at the L4 level. Table 1 offers a comparison of predicted CSF 163 flow parameters with our MR measurements.

164 The pressure and volumetric flow results of the massive supercomputing simulations 165 were fitted into a comprehensive CSF pressure and volumetric flow map condensing all 166 computational results into an easy-to-use Fourier-approximation formula. The approximations 167 formulae in eqs 5-8, with coefficients given in electronic supplemental material Supplemental 168 Table 2, permit the calculation of the pressure and flow waveforms for the specific subject for 169 any point in the spine and time point in the cardiac cycle. Approximations for location, $l \subset\{\mathrm{CM}$, $170 \mathrm{C} 4, \mathrm{~T} 1, \mathrm{~T} 6, \mathrm{~T} 11, \mathrm{~L} 4, \mathrm{~S} 3\}$ along the spine are linearly interpolated between two bounding levels $171\left(l_{\mathrm{i}}, l_{\mathrm{i}+1}\right)$ using a weighting factor, $\omega$. Fig. 5 shows a two-dimensional map of volumetric flow rate, $172 V(l, t)$, and pressure, $P(l, t)$, dynamics as function of spinal location, $l$, and time, t. These maps 173 approximate the experimental and computational results with continuous functions, and can be 174 used to calculate the precise timing, phase lags, and amplitudes of the pressure and fluid flow 175 pulsations in the spinal CSF.

$$
V l, t=\omega l \text { flit }+-\omega l \text { fli }+t
$$

$$
P l, t=\omega l f l i t+-\omega l f l i+t
$$

$$
\omega i l=l i+1-l l i+1-l i \quad l i<l<l i+10
$$

else

$$
\text { fit }=a l i \sin n t+b l i \cos n t
$$
volumetric flow rate map is $0.84 \%$ and for the pressure map is $0.66 \%$. These results show that 
184 the maps perfectly reproduce the expensive simulation results. Fig. 6 offers a detailed comparison of peak pressure magnitudes in systole and diastole for three data sets: (i) MRI data, (ii) CFD simulated values, and (iii) Fourier approximation at each plane of interest. Again the easy-to-use Fourier map encodes the MRI measurement and simulations with high fidelity.

\subsection{Nerve root influence on micro-flow patterns in the full SAS model}

Next, microcirculatory flow patterns of the full CNS model were characterized. Previous CFD simulations in an idealized spinal SAS without nerve roots already showed that pulsations break laminar fluid layers. ${ }^{14}$ Here, CNS wide simulations with all 31 nerve root pairs induced even more vigorous mixing in the spinal CSF and introduced eddies with recirculation zones. Typical micromixing patterns generated by nerve roots are depicted in Fig. 7. At peak systole frames $\mathrm{A}, \mathrm{C}, \mathrm{E}$ visualize regions of recirculation against laminar bulk flow around the base of nerve roots at $\mathrm{C} 4, \mathrm{~T} 6$, and $\mathrm{L} 4$, respectively. These micromixing patterns and recirculation zones are driven by the pulsating CSF flow around the nerve roots which act as baffles. ${ }^{23}$ Complex flow profiles in the cranial SAS are visualized in Appendix 3. To characterize the rotational component of the fluid flow, the vorticity defined as the magnitude of the curl of the velocity vector flow field in eq 6 was computed.

$$
\text { Vorticity }=\nabla \times v
$$

Streamlines and vorticity isosurfaces illustrate regions of high vorticity adjacent to nerve roots in the spinal SAS in Fig. 7. Frames B,D,F characterize the location and size of the recirculation zones. Around the $\mathrm{C} 4$ nerve root, a vortex forms with core diameter, $\mathrm{d}=0.33 \mathrm{~cm}$, and spatial extension of $0.036 \mathrm{~cm}^{3}$. The vortex at $\mathrm{T} 6$ region is the largest with $\mathrm{d}=0.56 \mathrm{~cm}$ with a volume of $0.176 \mathrm{~m}^{3}$. For the L4 region, the vortex is $\mathrm{d}=0.13 \mathrm{~cm}$ its volume is $0.0022 \mathrm{~cm}^{3}$. Local macroscopic Reynolds numbers were computed at each plane with eq. 12 (Appendix 1.6), 
$208 \mathrm{Re}=112$ at $\mathrm{C} 4,<86$ at $\mathrm{T} 6,<67$ at $\mathrm{L} 4$. It is remarkable that vortices occur, despite the low

209 Reynolds number. These patterns are known as geometry-induced flow. ${ }^{30}$

2103.3 Effect of trabeculae on flow resistance and micromixing profiles

211 The impact of trabeculae on CSF flow resistance was estimated with the help of detailed

212 CFD simulations. The density of arachnoid trabeculae throughout the spinal SAS is not well

213 characterized. Based on canine data, ${ }^{4,35}$ Stockman arrives at a conservative estimate of

21424 trabeculae in the dorsal and ventral regions of an idealized spinal model; this equals about

21585 trabeculae per $\mathrm{cm}^{3}\left(\mathrm{Tr}^{-3}\right)$. To cover a broad spectrum of physiologically relevant trabeculae

216 densities, we artificially introduced between $40-200 \mathrm{Tr} \mathrm{cm}^{-3}$ of CSF into the computational mesh

217 of spinal segments. To demonstrate mesh independence, pressure and velocity magnitude along a

218 ray through the spinal segment mesh are plotted in Fig. 8D for different mesh densities of $12 \mathrm{~m}$,

$21922 \mathrm{~m}$, and $54 \mathrm{~m}$. Mesh independence for pressure and velocity was studied with no trabeculae

220 (data not shown) and with $100 \mathrm{Tr} \mathrm{cm}-3$ meshes. For the $54 \mathrm{~m} \mathrm{mesh}$ with $40 \mathrm{Tr} \mathrm{cm}^{-3}$, the pressure

221 increases from 58 to $86 \mathrm{~Pa} ; 100 \mathrm{Tr} \mathrm{cm}^{-3}$ trabeculae 58 to $109 \mathrm{~Pa} ; 200 \mathrm{Tr} \mathrm{cm}^{-3} 58$ to $145 \mathrm{~Pa}$. The

222 average pressure drop that develops across the spinal mesh segments is graphically represented

223 in Fig. 8B, as a function of number of trabeculae per $\mathrm{cm}^{-3}(\mathrm{~N})$ given by the following formula:

$$
\Delta P=0.5064 * N+64.64
$$

Pressure contours across the segment are given in Fig. 8A. The fine trabeculae

226 simulations with a $54 \mathrm{~m}$ mesh typically converged in one to two days of using 128 parallel 227 processors and consuming a total of $1400 \mathrm{CPU}$ hours on the Blacklight Supercomputing Facility.

In summary, the pressure drop simulation results suggest that trabeculae significantly

229 increase the CSF resistance, almost double compared to idealized CSF compartments without 
230 trabeculae for a physiologically consistent estimate of 100 trabeculae $\mathrm{cm}^{-3}$. For the

231200 trabeculae $\mathrm{cm}^{-3}$ mesh, the resistance would increase by a factor as great as 2.5 .

In addition to elevating flow resistance, arachnoid trabeculae further enhance flow

233 recirculation. Isosurfaces using the vorticity threshold $<6 \mathrm{~s}^{-1}$ are generated to visualize vortex

234 phenomena in Fig. 8C. With microanatomy, there is strong recirculation around trabeculae. In

235 the model lacking microanatomy, vorticity is minimal.

236 3.4 The effect of CSF dynamics on intrathecally administered drug transport.

237 The flow simulations already demonstrated geometry-induced micromixing with vortex

238 generation. These counter-currents in the spinal CSF flow are a significant finding clinically,

239 because they drastically impact drug biodistribution in the CNS. Fig. 9A visualizes drug

240 distribution contours in the full spinal SAS for the first 30 minutes. Drug concentration is plotted

241 over time at spinal regions (C4, T6, L4) in Fig. 9B-D for the first 300 minutes. Peak drug

242 concentration in the CSF occurs at $31 \mathrm{~min}$ for L4, $64 \mathrm{~min}$ for T6, and $237 \mathrm{~min}$ at C4. To test the

243 role of CSF bulk flow on drug dispersion, we also performed simulations without bulk flow. The

244 casual motion of the drug was almost as fast as in the presence of bulk flow, indicating that CSF

245 bulk flow does not affect the cranial upward motion of IT delivered drugs. These results allow

246 the important conclusion that the open question regarding the classical or more recent CSF

247 reabsorption theories ${ }^{15,28}$ would not critically affect the dispersion speed of CSF administered

248 drugs. The simulations confirm our prior predictions implicating CSF pulse and frequency as the

249 main driving forces of solute dispersion in slow continuous CSF drug administration. ${ }^{12,14}$

250 Additionally drug motion is simulated on a spinal SAS without nerve roots and peak drug

251 concentrations occur at $62 \mathrm{~min}$ for L4, 89 min for T6, and $285 \mathrm{~min}$ for C4. Drug motion in the 
252 caudocranial direction has a slower distribution in the absence of nerve roots. These three comparative simulations of drug dispersion indicate that nerve roots facilitate CSF mixing.

We also studied the effect of molecular diffusivity on the speed of caudal drug motion. Simulations with different molecular diffusivities to mimic different solutes showed that drug dispersion speed was almost the same in all runs (computations not shown). The speed of the cranial motion for a CSF stroke volume of $0.76 \mathrm{~mL}$ and a pulse frequency of $1 \mathrm{~Hz}$ was about $0.21 \mathrm{~cm} \mathrm{~min}^{-1}$, irrespective of the Diffusion coefficient. The computational results confirm that drug biodistribution depends strongly on CSF pulsatility, but only weakly on drug diffusivity. The reported cranial advance of the drug front agrees with clinical studies of lumbar drug infusion, which observed the drug front advancing at a rate of $0.16-0.31 \mathrm{~cm} \mathrm{~min}^{-1} .^{27,33}$

\section{Discussion}

This computational study aimed at predicting the CSF dynamics in a subject-specific model of the entire CNS including microanatomical aspects. The massive CFD computations assessed the effect of microanatomy on CSF flow and flow resistance. Simulations found the caudal flow phase to last $20-30 \%$ of the cardiac cycle, which agreed with MRI measurements. The cisterna magna had a stroke volume of $0.76 \mathrm{~mL}$ with caudal decrease in amplitudes.

Pressure pulsatility along the spinal canal was characterized. The craniocaudal pressure wave was sharp and short in systole, followed by a reversal in the slower diastolic phase. Substantial phase lags between pressure and velocity waves along the spinal column were predicted consistent with the spinal Womersley numbers range, $8<\alpha<16$. Spectral fitting of the simulated pressure and flow signals at different vertebral levels yields a closed formula for the pressure and mean volumetric flow waves for any point in the spinal SAS or time point in the cardiac cycle. The volumetric flow and the pressure maps are easy to use and encode 
275 experiments and CFD simulations with high fidelity. The approximation formulae also enable 276 easy computation of the amplitude attenuation, as well as phase lags between the pressure and 277 velocity wave peaks.

278 Nerve roots were found to create substantial obstacles to the pulsating CSF flow that 279 break the laminar fluid layers and generate geometry-induced flow profiles despite the relatively 280 low Reynolds numbers, $\operatorname{Re}<179$. The mixing effects in the spine are similar to the mixing effects 281 observed in pulsatile flows in baffled cylinders as described elsewhere. ${ }^{23}$ The extent and intensity 282 of vortex formation was visualized adjacent to nerve roots and the cerebral aqueduct for all 283 phases of the cardiac cycle.

284 Our simulation results permit the estimation of flow resistance attributable to 285 microanatomy. In the densest case, $200 \mathrm{Tr} \mathrm{cm}^{-3}$ the pressure drop experienced a 2.5 -fold 286 increase. For the physiologically more likely range of $100 \mathrm{Tr} \mathrm{cm}^{-3}$, the flow resistance almost 287 doubled. Our results and Gupta's work ${ }^{9}$ agree in identifying trabeculae as major sources of flow 288 resistance. Computations of expected pressure ranges are useful to guide experiments to validate 289 the predicted impact of microanatomical aspects.

290 Significance of CNS-wide CSF simulations. In this report, we addressed the lack of 291 microanatomical detail in prior CFD models by artificially augmenting a subject-specific mesh 292 with the computer-generated microanatomical aspects. Clearly, the statistical, coarse 293 representation of the microstructures suggested here does not necessarily match subject-specific 294 properties, but yields quantitative results on the sensitivity of pulsatile flow patterns and 295 geometry-induced flow resistance due to presence of microscopic obstacles. Although we could 296 at this point not ascertain experimentally the exact micro-geometry of the spinal SAS, the 297 computational results support findings by prior authors for the need to include these structures in 
298 future spinal CFD simulation studies to achieve more realistic approximations of pressure drops 299 and subject-specific flow measurements. The more than two-fold increase in flow resistance over 300 an ideal spinal SAS model without microanatomy predicted in this study may also explain the 301 substantial discrepancies between models and experiments in earlier studies. A second perspective underscoring the significance of CNS-wide simulation is related to 303 the open question regarding the motor of CSF motion. The exact source location and type of 304 force coupling between the expanding and contracting vasculature, brain movement and induced 305 CSF dynamics are uncertain. The present model of the entire CNS system completely enclosing 306 all CSF-filled spaces for a specific subject is a first step in the direction of linking vascular 307 pulsations to CSF dynamics. Results such as the phase lags which can easily be computed from 308 the volumetric flow and pressure maps for any point in time or location along the spinal cord will 309 be needed to trace the CSF pulse waves back to the main arteries or volumetric dilatations of the 310 microcirculatory bed that causes them.

311 Finally, the CNS wide model enabled us to shed light on the role of CSF bulk flow in 312 drug transport. Recently, the classical hypothesis of CSF production in the choroid causing a net 313 bulk CSF flow followed by reabsorption to the veins of the superior sagittal sinuses through the 314 arachnoid villi has been questioned. ${ }^{28}$ An argument in support of the classical CSF production 315 and reabsorption hypothesis held that the caudocranial transport of intrathecally administered 316 drugs as observed in the spinal anesthesia or the treatment of spasticity was evidence for CSF 317 bulk flow. MR flow measurements are too inaccurate to ascertain the extent of CSF bulk flow, 318 yet comparative simulations with and without the assumption of bulk flow, reveal that cranial 319 drug transport does occur rapidly due to CSF pulsations even without net CSF bulk flow. Also 320 solute diffusivity is a minor factor for the speed of solute dispersion. We demonstrated that the 
321 pulse frequency and CSF flow amplitude transmitted from the blood to the CSF is a significant

322 factor determining the speed of drug dispersion.

\section{Conclusions and Limitations}

The dynamic 3D model presented above is a first attempt to predict CSF circulation in carefully quantified and encoded in a comprehensive Fourier Map. The microcirculatory flow pattern introduced by nerve roots and arachnoid trabeculae were characterized with the use of massively parallel supercomputer simulations. support their role as enhancers of micromixing, despite the low Reynolds numbers of the CSF

331 dynamics. It is reasonable to conjecture that trabeculae in pulsatile CSF flow prevent

332 bioaccumulation of debris and metabolites, thus protecting sensitive nerve roots and neurons.

333 The proposed methods were shown to predict biodistribution of drugs after intrathecal infusion;

334 rapid drug dispersion was linked to convection facilitated by eddies and vortices induced into the 335 pulsatile CSF flow by spinal microanatomical aspects.

336 Limitations. Since the actual source locations of the vascular-parenchyma-CSF

337 interaction are not yet known, the cerebro-vascular bed was not explicitly modeled in this study.

338 Due to the uncertainty pertaining to the biomechanical properties of the CNS, especially the 339 deformability of dura and epidural spaces, we implemented explicit boundary motion instead of 340 attempting to predict wall deformation, although methods described previously by Sweetman for 341 full two-way implicit fluid structure interaction could be adopted. ${ }^{37}$ A future, more complete,

342 model of CSF dynamics could incorporate organ-wide reconstruction of the vasculature ${ }^{20}$ in 
343 contact with the CSF and brain parenchyma. In the drug transport simulations, the injection

344 impulse was neglected due to the slow micro-infusion volumes used.

345 In this first full model of the full CNS, we also did not perform multi-subject validation

346 of the CSF flow field, because the emphasis lay on the characterization of parameter sensitivity

347 as a function of trabecular density. A multi-individual study would require computational meshes

348 for each subject; and IT drug infusions cannot be performed on healthy volunteers. Therefore we

349 limited the scope to a detailed analysis of one subject, but with parametric variation of trabecular

350 density and random orientation compared to flows in spinal SAS without microanatomy.

351 Multiple subject validation and inclusion of patients suffering from a disease exceeded the scope

352 and focus of our computational study here, but should be considered in a future study.

353 Velocity measurements were acquired using conventional MRI scans conducted on a

354 volunteer in the supine position, therefore variations of body position are not accounted for in

355 this comprehensive simulation of the entire CSF space, although it is known that posture 356 influences intracranial compliance ${ }^{1}$.

\section{Acknowledgements}

360 The authors would like to gratefully acknowledge partial support from the National Science 361 Foundation grant CBET-0756154. This work used the Extreme Science and Engineering 362 Discovery Environment (XSEDE), which is supported by National Science Foundation 363 grant number OCI-1053575. Specifically, it used the Blacklight system at the Pittsburgh

364 Supercomputing Center (PSC). We would like to thank C. Wivel from Materialize Inc. for 365 providing a research license for Mimics image reconstruction software. M. Hettiarachchi, 
work.

\section{References}

1. Alperin, N., S. G. Hushek, S. H. Lee, A. Sivaramakrishnan, and T. Lichtor. MRI study of cerebral blood flow and CSF flow dynamics in an upright posture: the effect of posture on the intracranial compliance and pressure. Acta Neurochir. Suppl. 95:177-181, 2005.

2. Alperin, N., A. Sivaramakrishnan, and T. Lichtor. Magnetic resonance imaging-based measurements of cerebrospinal fluid and blood flow as indicators of intracranial compliance in patients with Chiari malformation. J. Neurosurg. 103:46-52, 2005.

3. Bhadelia, R. A., A. R. Bogdan, R. F. Kaplan, and S. M. Wolpert. Cerebrospinal fluid pulsation amplitude and its quantitative relationship to cerebral blood flow pulsations: a phase-contrast MR flow imaging study. Neuroradiology 39:258-264, 1997.

4. Cloyd, M. W., and F. N. Low. Scanning electron microscopy of the subarachnoid space in the dog. I. Spinal cord levels. J. Comp. Neurol. 153:325-367, 1974.

5. Dumoulin, C. L., S. P. Souza, M. F. Walker, and E. Yoshitome. Time-resolved magnetic resonance angiography. Magn. Reson. Med. 6:275-286, 1988.

6. Enzmann, D. R., and N. J. Pelc. Brain motion: measurement with phase-contrast MR imaging. Radiology 185:653-660, 1992.

7. Gupta, S., and D. Poulikakos. Analytical solution for pulsatile viscous flow in a straight elliptic annulus and application to the motion of the cerebrospinal fluid. Phys. Fluids - PHYS FLUIDS 20:, 2008.

8. Gupta, S., M. Soellinger, P. Boesiger, D. Poulikakos, and V. Kurtcuoglu. Three-dimensional computational modeling of subject-specific cerebrospinal fluid flow in the subarachnoid space. $J$. Biomech. Eng. 131:021010, 2009.

9. Gupta, S., M. Soellinger, D. M. Grzybowski, P. Boesiger, J. Biddiscombe, D. Poulikakos, and V. Kurtcuoglu. Cerebrospinal fluid dynamics in the human cranial subarachnoid space: an overlooked mediator of cerebral disease. I. Computational model. J. R. Soc. Interface R. Soc. 7:1195-1204, 2010.

10. Haller, F. R., and F. N. Low. The fine structure of the peripheral nerve root sheath in the subarachnoid space in the rat and other laboratory animals. Am. J. Anat. 131:1-19, 1971.

11. Heidari Pahlavian, S., T. Yiallourou, R. S. Tubbs, A. C. Bunck, F. Loth, M. Goodin, M. Raisee, and B. A. Martin. The Impact of Spinal Cord Nerve Roots and Denticulate Ligaments on Cerebrospinal Fluid Dynamics in the Cervical Spine. PLoS ONE 9:e91888, 2014.

12. Hettiarachchi, H. D. M., Y. Hsu, T. J. Harris Jr, R. Penn, and A. A. Linninger. The effect of pulsatile flow on intrathecal drug delivery in the spinal canal. Ann. Biomed. Eng. 39:2592-2602, 2011. 
13. Hofmann, E., M. Warmuth-Metz, M. Bendszus, and L. Solymosi. Phase-Contrast MR Imaging of the Cervical CSF and Spinal Cord: Volumetric Motion Analysis in Patients with Chiari I Malformation. Am. J. Neuroradiol. 21:151-158, 2000.

14. Hsu, Y. B., H. D. M. Hettiarachchi, D. C. Zhu, and A. A. Linninger. The Frequency and Magnitude of Cerebrospinal Fluid Pulsations Influence Intrathecal Drug Distribution: Key Factors for Interpatient Variability. [Miscellaneous Article]. Anesth. Analg. August 2012 115:386-394, 2012.

15. Klarica, M., B. Miše, A. Vladić, M. Radoš, and D. Orešković. "Compensated hyperosmolarity" of cerebrospinal fluid and the development of hydrocephalus. Neuroscience 248:278-289, 2013.

16. Kurtcuoglu, V., D. Poulikakos, and Y. Ventikos. Computational modeling of the mechanical behavior of the cerebrospinal fluid system. J. Biomech. Eng. 127:264-269, 2005.

17. Kurtcuoglu, V., M. Soellinger, P. Summers, K. Boomsma, D. Poulikakos, P. Boesiger, and Y. Ventikos. Computational investigation of subject-specific cerebrospinal fluid flow in the third ventricle and aqueduct of Sylvius. J. Biomech. 40:1235-1245, 2007.

18. Laitinen, L. Origin of Arterial Pulsation of Cerebrospinal Fluid. Acta Neurol. Scand. 44:168-176, 1968.

19. Linge, S. O., V. Haughton, A. E. Løvgren, K. A. Mardal, and H. P. Langtangen. CSF flow dynamics at the craniovertebral junction studied with an idealized model of the subarachnoid space and computational flow analysis. AJNR Am. J. Neuroradiol. 31:185-192, 2010.

20. Linninger, A. A., B. Sweetman, and R. Penn. Normal and hydrocephalic brain dynamics: the role of reduced cerebrospinal fluid reabsorption in ventricular enlargement. Ann. Biomed. Eng. 37:14341447, 2009.

21. Linninger, A. A., M. Xenos, D. C. Zhu, M. R. Somayaji, S. Kondapalli, and R. D. Penn. Cerebrospinal fluid flow in the normal and hydrocephalic human brain. IEEE Trans. Biomed. Eng. 54:291-302, 2007.

22. Loth, F., M. A. Yardimci, and N. Alperin. Hydrodynamic Modeling of Cerebrospinal Fluid Motion Within the Spinal Cavity. J. Biomech. Eng. 123:71-79, 2000.

23. Mackley, M. R., and X. Ni. Mixing and dispersion in a baffled tube for steady laminar and pulsatile flow. Chem. Eng. Sci. 46:3139-3151, 1991.

24. Marmarou, A., K. Shulman, and J. LaMorgese. Compartmental analysis of compliance and outflow resistance of the cerebrospinal fluid system. J. Neurosurg. 43:523-534, 1975.

25. Marmarou, A., K. Shulman, and R. M. Rosende. A nonlinear analysis of the cerebrospinal fluid system and intracranial pressure dynamics. J. Neurosurg. 48:332-344, 1978.

26. Martin, B. A., W. Kalata, N. Shaffer, P. Fischer, M. Luciano, and F. Loth. Hydrodynamic and Longitudinal Impedance Analysis of Cerebrospinal Fluid Dynamics at the Craniovertebral Junction in Type I Chiari Malformation. PLoS ONE 8:e75335, 2013.

27. Meythaler, J. M., S. Guin-Renfroe, R. C. Brunner, and M. N. Hadley. Intrathecal Baclofen for Spastic Hypertonia From. Stroke 32:2099-2109, 2001.

28. Orešković, D., and M. Klarica. Development of hydrocephalus and classical hypothesis of cerebrospinal fluid hydrodynamics: Facts and illusions. Prog. Neurobiol. 94:238-258, 2011.

29. Pelc, N. J., M. A. Bernstein, A. Shimakawa, and G. H. Glover. Encoding strategies for three-direction phase-contrast MR imaging of flow. J. Magn. Reson. Imaging JMRI 1:405-413, 1991.

30. Piot, E., and S. Tavoularis. Gap instability of laminar flows in eccentric annular channels. Nucl. Eng. Des. 241:4615-4620, 2011.

31. Roldan, A., O. Wieben, V. Haughton, T. Osswald, and N. Chesler. Characterization of CSF hydrodynamics in the presence and absence of tonsillar ectopia by means of computational flow analysis. AJNR Am. J. Neuroradiol. 30:941-946, 2009.

32. Rutkowska, G., V. Haughton, S. Linge, and K.-A. Mardal. Patient-specific 3D simulation of cyclic CSF flow at the craniocervical region. AJNR Am. J. Neuroradiol. 33:1756-1762, 2012.

33. Shapiro, W. R., D. F. Young, and B. M. Mehta. Methotrexate: Distribution in Cerebrospinal Fluid after Intravenous, Ventricular and Lumbar Injections. N. Engl. J. Med. 293:161-166, 1975. 
34. Somayaji, M. R., M. Xenos, L. Zhang, M. Mekarski, and A. A. Linninger. Systematic design of drug delivery therapies. Comput. Chem. Eng. 32:89-98, 2008.

35. Stockman, H. W. Effect of Anatomical Fine Structure on the Flow of Cerebrospinal Fluid in the Spinal Subarachnoid Space. J. Biomech. Eng. 128:106-114, 2005.

36. Stockman, H. W. Effect of anatomical fine structure on the dispersion of solutes in the spinal subarachnoid space. J. Biomech. Eng. 129:666-675, 2007.

37. Sweetman, B. J. Cerebrospinal Fluid Flow in Normal and Hydrocephalic Brains. , 2012.at $<$ http://oatd.org/oatd/record?record=handle\%5C:10027\%5C\%2F8357>

38. Sweetman, B., and A. A. Linninger. Cerebrospinal fluid flow dynamics in the central nervous system. Ann. Biomed. Eng. 39:484-496, 2011.

39. Sweetman, B., M. Xenos, L. Zitella, and A. A. Linninger. Three-dimensional computational prediction of cerebrospinal fluid flow in the human brain. Comput. Biol. Med. 41:67-75, 2011.

40. Tain, R.-W., A. M. Bagci, B. L. Lam, E. M. Sklar, B. Ertl-Wagner, and N. Alperin. Determination of cranio-spinal canal compliance distribution by MRI: Methodology and early application in idiopathic intracranial hypertension. J. Magn. Reson. Imaging JMRI 34:1397-1404, 2011.

41. Towns, J., T. Cockerill, M. Dahan, I. Foster, K. Gaither, A. Grimshaw, V. Hazlewood, S. Lathrop, D. Lifka, G. D. Peterson, R. Roskies, J. R. Scott, and N. Wilkens-Diehr. XSEDE: Accelerating Scientific Discovery. Comput. Sci. Eng. 16:62-74, 2014.

42. Wagshul, M. E., J. J. Chen, M. R. Egnor, E. J. McCormack, and P. E. Roche. Amplitude and phase of cerebrospinal fluid pulsations: experimental studies and review of the literature. J. Neurosurg. 104:810-819, 2006.

43. Weller, R. O. Microscopic morphology and histology of the human meninges. Morphol. Bull. Assoc. Anat. 89:22-34, 2005.

44. Womersley, J. R. Method for the calculation of velocity, rate of flow and viscous drag in arteries when the pressure gradient is known. J. Physiol. 127:553-563, 1955.

45. Yiallourou, T. I., J. R. Kröger, N. Stergiopulos, D. Maintz, B. A. Martin, and A. C. Bunck. Comparison of 4D Phase-Contrast MRI Flow Measurements to Computational Fluid Dynamics Simulations of Cerebrospinal Fluid Motion in the Cervical Spine. PLoS ONE 7:e52284, 2012.

46. Zhu, D. C., M. Xenos, A. A. Linninger, and R. D. Penn. Dynamics of lateral ventricle and cerebrospinal fluid in normal and hydrocephalic brains. J. Magn. Reson. Imaging JMRI 24:756-770, 2006. 
Figure 1: Presentation of the microanatomical structures present in the spinal mesh models. (A) An electron microscope image and a schematic, adapted from Weller, ${ }^{42}$ captures trabeculae (Tr) bridging the arachnoid (Ar) and pia (P) mater and illustrated the uniform distribution of trabeculae throughout the SAS. B-D) Overview of the process for artificially generating arachnoid trabeculae within a short cervical spinal segment. (B) A short spinal segment is cropped and tubes are projected through the volume mesh at random levels along the $\mathrm{Y}$-axis and angles in the XZ-plane. (C) Volume elements intersected by the analytical tubes are deleted from the mesh and neighboring faces grouped together as a boundary. (D) The final volume mesh with 100 trabeculae and two pairs of nerve roots has $54 \mathrm{~m}$ elements. E-G. Details of the microantomical aspects in the subject-specific computational mesh. (E) An axial cross section of the SAS at the level of a nerve root. (F) A thoracolumbar segment shows five nerve root pairs branching off the spinal cord and extending through the SAS to the dura membrane. $(\mathrm{G})$ A magnified visualization of the subject-specific geometry of the nerve pairs.

Table 1: Summary of Simulated and MR measured Flow Parameters

Figure 2: Velocity profiles computed for axial plane in regions of interest (CM, C1, C4, C7, T1, T4, T6, T9, T11, L2, L4, and S1) in different phases of the cardiac cycle (CC). Velocity scale is identical for all planes and time points of the CC. Red encodes the craniocaudal flow direction, blue represents caudocranial flow, and green signals almost stagnant flow $\left(\|\mathrm{v}\|<0.1 \mathrm{~cm} \mathrm{~s}^{-1}\right)$. The spinal cord terminates at the L2 level; below this level the peripheral nerves occupy the lower spinal subarachnoidal space.

Figure 3: Relationship of pressure and volumetric flow waves. The systole (red) has a high amplitude downward flow, the caudocranial recoil in the diastole (blue) is longer with slower flow. The amplitude of both pressure and volumetric flow rate gradually decrease from the Cisterna to the Sacral region. Also the phase lag increases in the craniocaudal direction.

Figure 4: Comparison of CSF flow between simulated and MRI measured data in the human spinal canal. An axial CINE-MRI contour of the CSF space identifies the imaging plane for the volumetric flow comparison. CSF flow is simulated on a subject-specific full CNS model reconstructed from T2 MRI data. Simulated volumetric flow profiles (solid curve) are overlaid with MRI derived volumetric flow profiles (solid line). Negative velocity (red) values signify craniocaudal flow during systole, positive (blue) values are the opposite direction.

Figure 5: Volumetric flow map and pressure map across the length of the spinal canal over the full cardiac cycle (CC) from Fourier approximations. Craniocaudal flow during systole is represented as negative velocities (red), while caudocranial flow during diastole is positive (blue), and green encodes stagnation $\left(\|\mathrm{v}\|<0.1 \mathrm{~cm} \mathrm{~s}^{-1} ; \mathrm{P}<1 \mathrm{~Pa}\right)$. The amplitudes of both flow and pressure decrease along the spinal SAS with the greatest magnitudes being superior at the cisterna magna (CM). Formulas for the Fourier approximations with the coefficients are given in Supplementary Table 1 in the appendix.

Figure 6: Peak pressure and stroke volume from the MR measurements, the CFD simulation, and the Fourier approximations show close agreement in timing and magnitude. Hence, the Fourier approximation is an excellent fit for the experimental and simulated data.

Figure 7: Visualization of complex micro-flow induced patterns in the spinal SAS. Velocity streamlines with magnifications show vortices at the C4, T6, and L4 regions. Streamline are color coded from 0 (blue) to 0.04 (red) $\mathrm{m} \mathrm{s}^{-1}$. Vortex sizes are delineated by isosurfaces. Despite the low Re number, geometry induced flow patterns are not laminar. Magnified vortex views show microcirculatory eddies forming close to the nerve roots.

Figure 8: Pressure drop and vorticity due to trabeculae. (A) Pressure contours for spinal SAS segment models with 40-200 $\mathrm{Tr} \mathrm{cm}^{-3}$. (B) The pressure drop increases with increasing number of trabeculae. Also 
simulations with different mesh density demonstrate mesh independence of pressure computations. (C) The vorticity is high around trabeculae, but almost absent in a computational mesh with no trabeculae (vorticity threshold $=6 \mathrm{~s}^{-1}$ ) (D) Mesh independence is demonstrated along a ray for different mesh densities. For the meshes with $22 \mathrm{~m}$ and $54 \mathrm{~m}$ elements, the computed pressure and velocity magnitudes are virtually identical.

Figure 9: Predicted drug distribution profiles over time for an IT injection in the CNS. (A) The timely evolution of the drug concentration profile shows a caudocranial rise of the injected drug. CSF Bulk flow due to arachnoid absorption did not significantly alter the speed of rostral drug motion. A one minute bolus injection is applied in a model with nerves (B) and without bulk flow (C) and an idealized annular model (D). Simulations in idealized annular models without micro-anatomy showed a significantly slower drug rise towards the cranial compartment. 

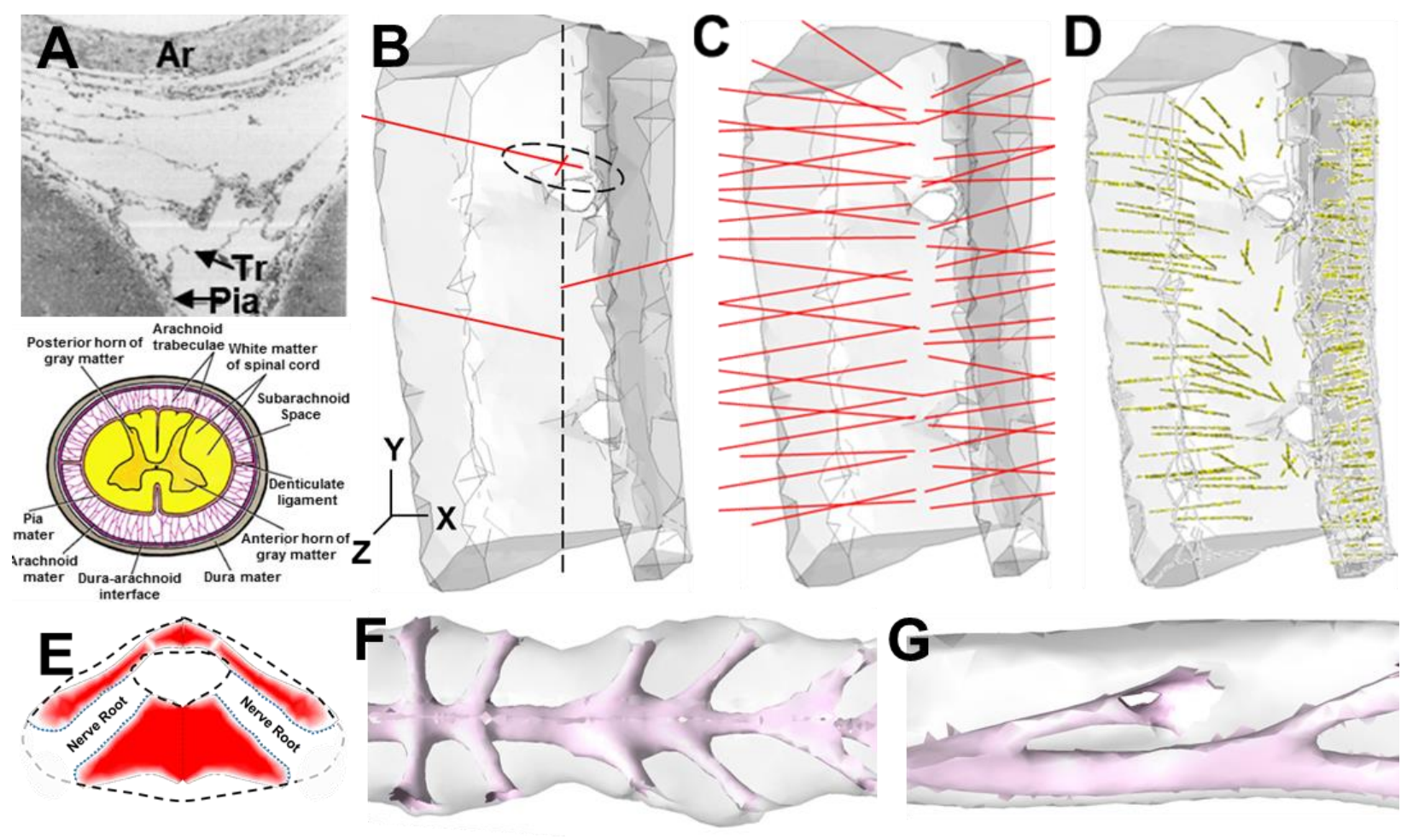


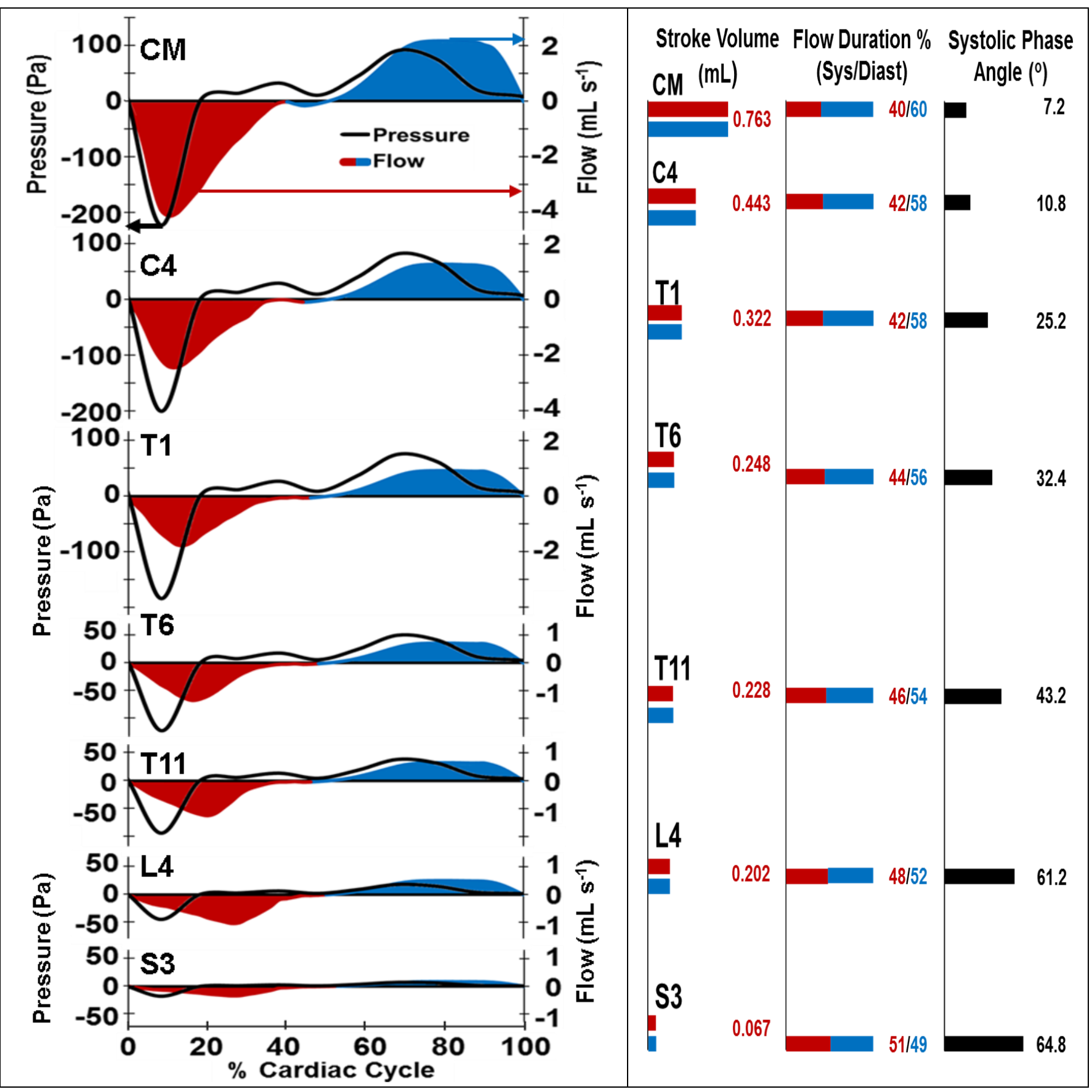




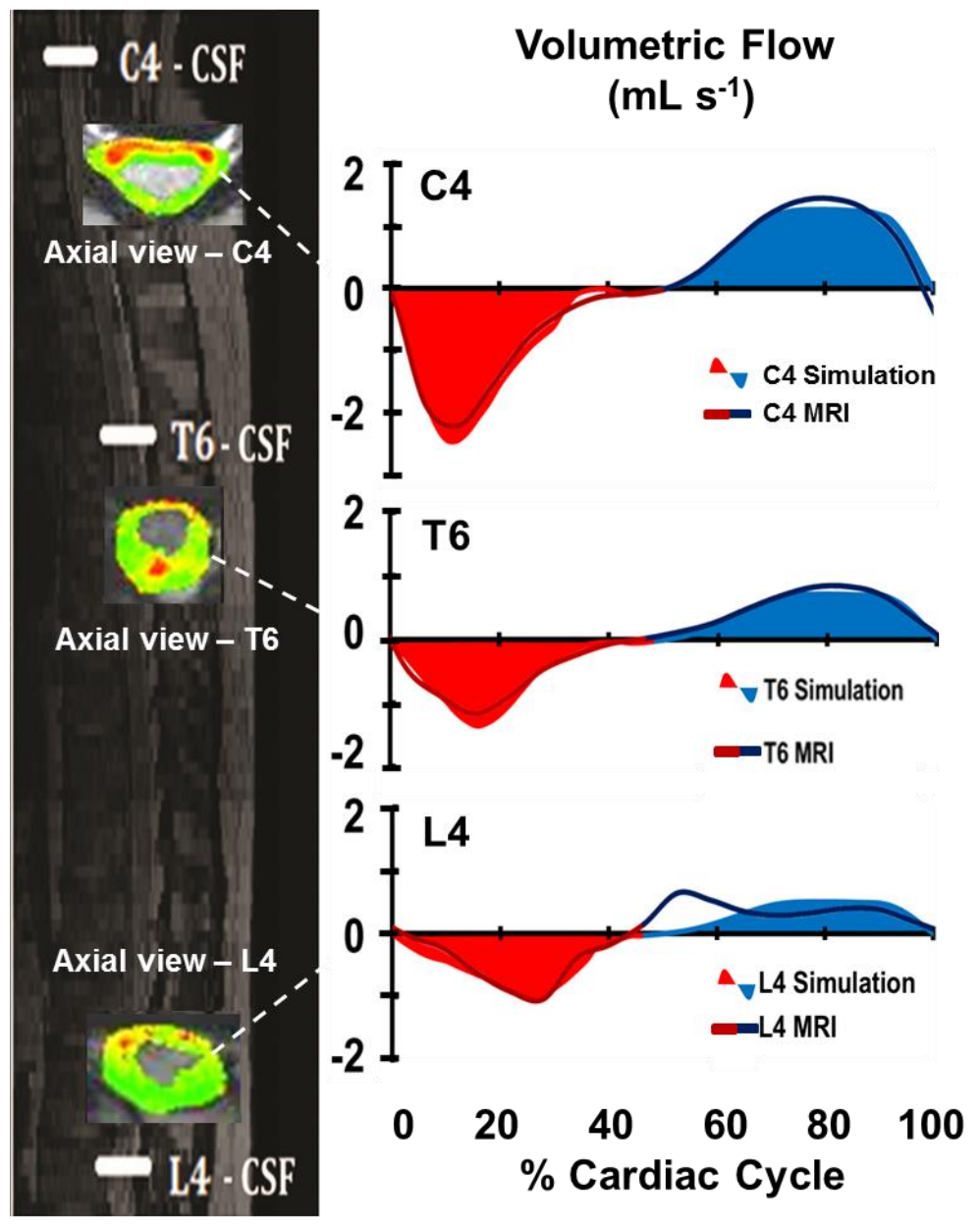

C4.CSF

metric Flow

$\left(\mathrm{mL} \mathrm{s}{ }^{-1}\right)$

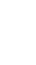

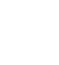




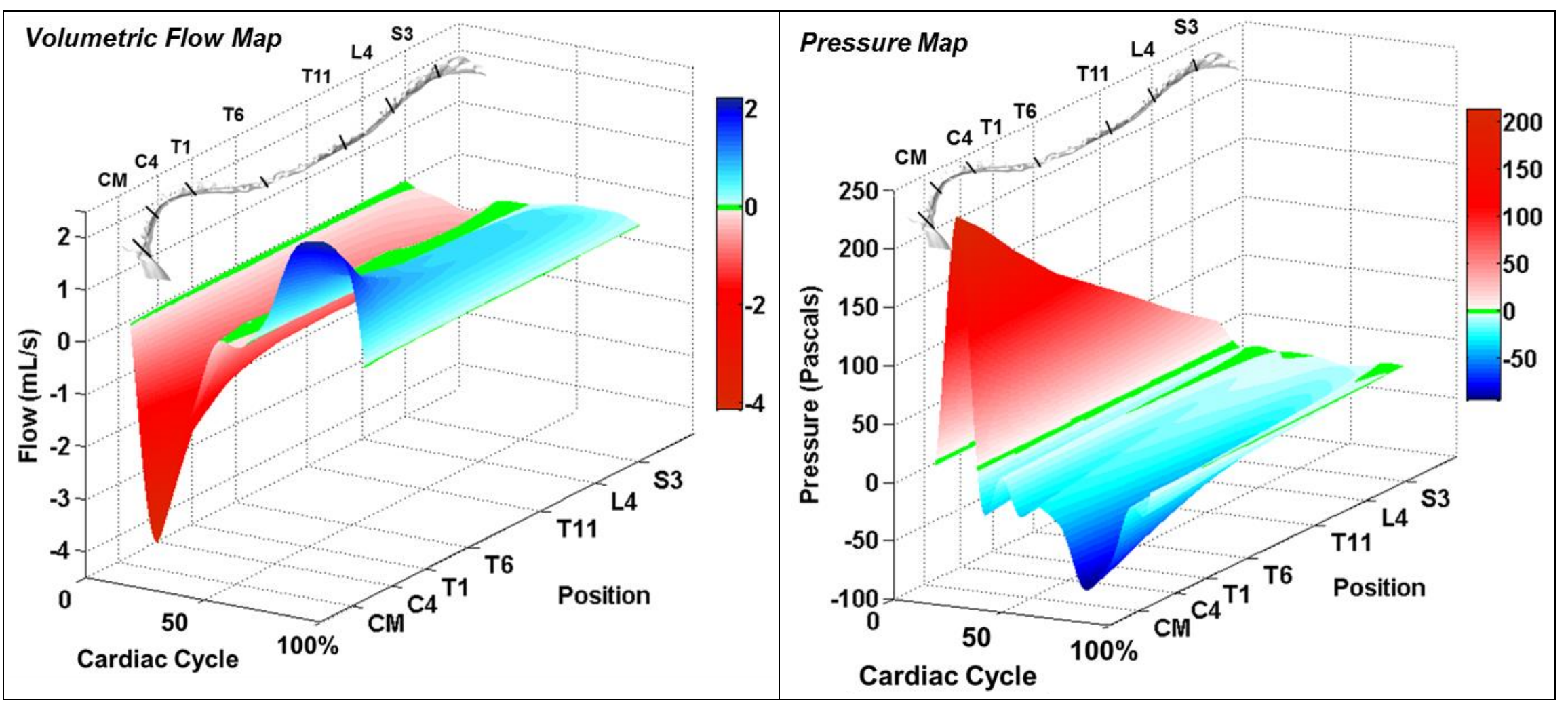




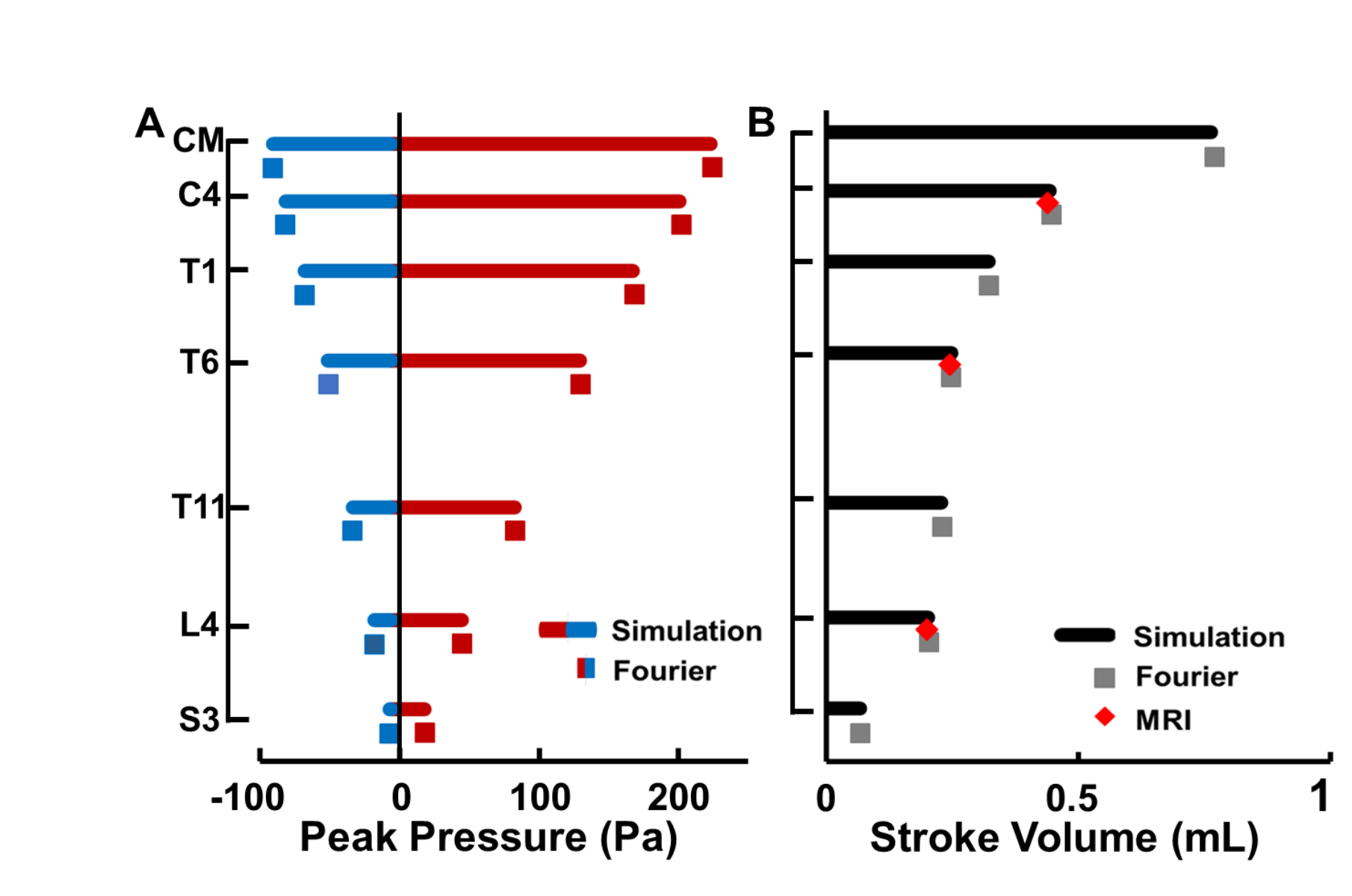

Figure 6

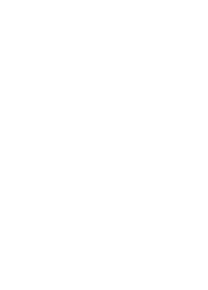

.

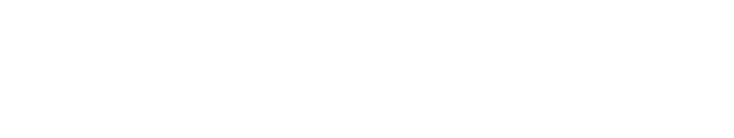

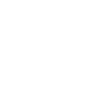




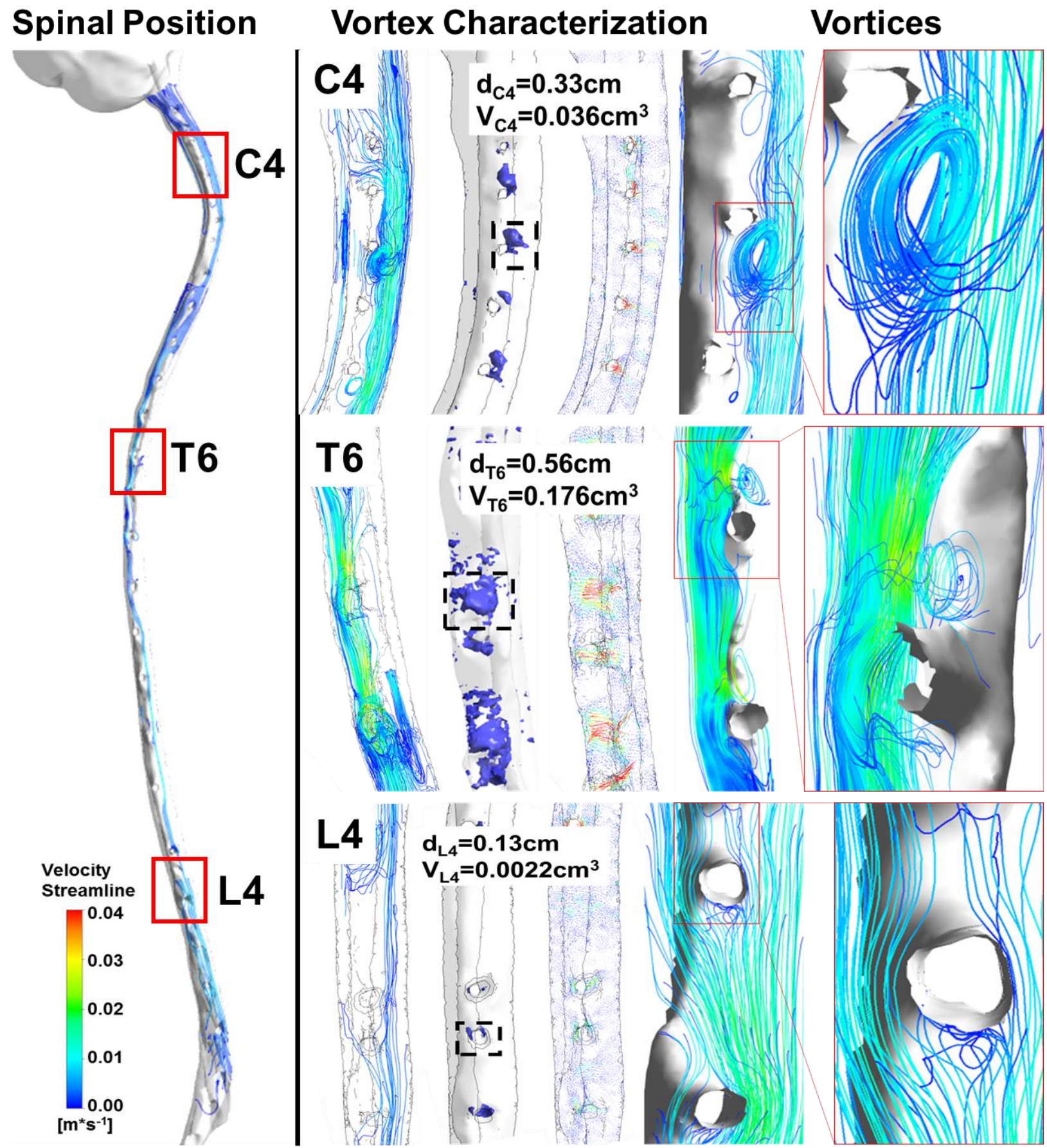




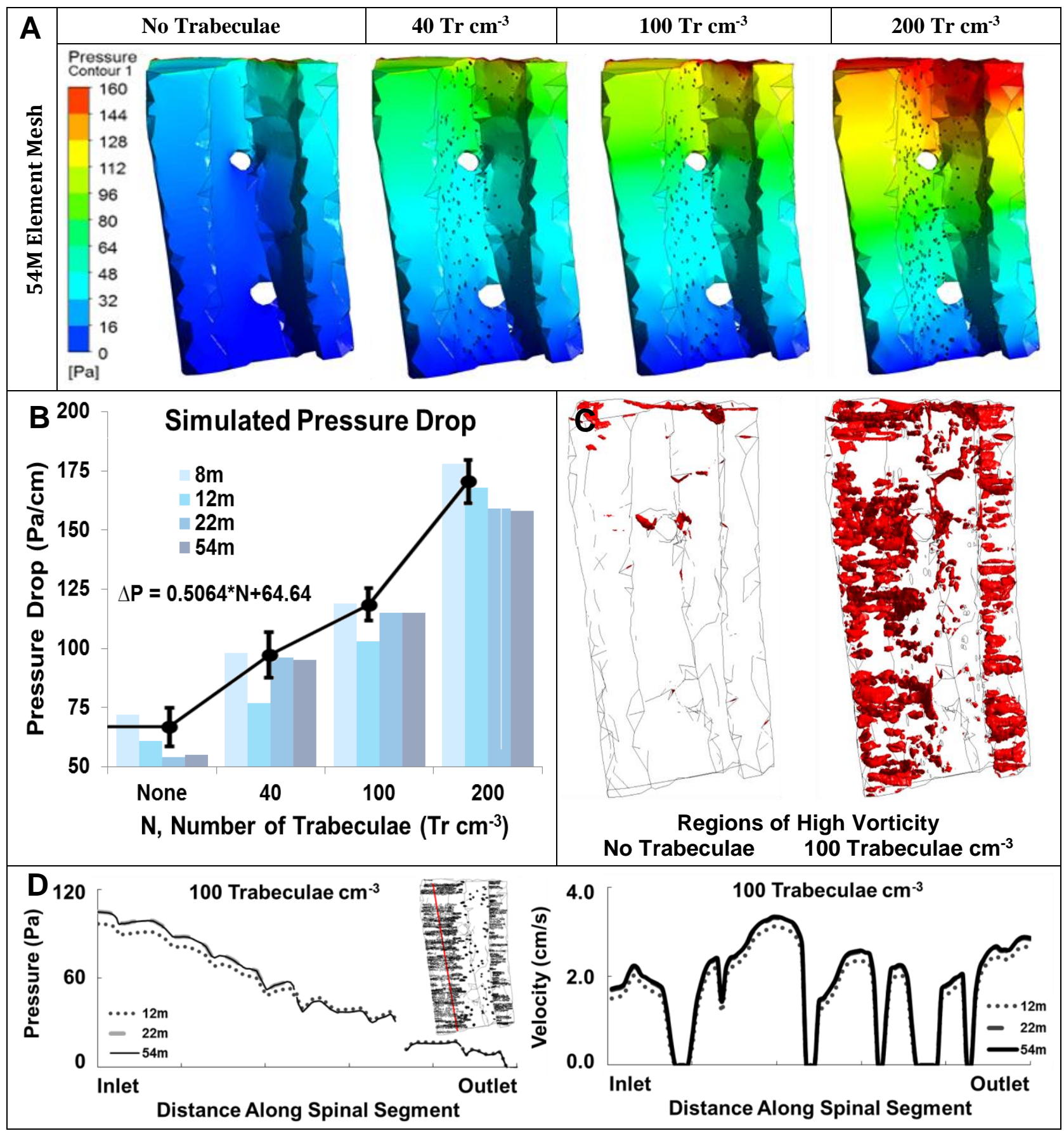



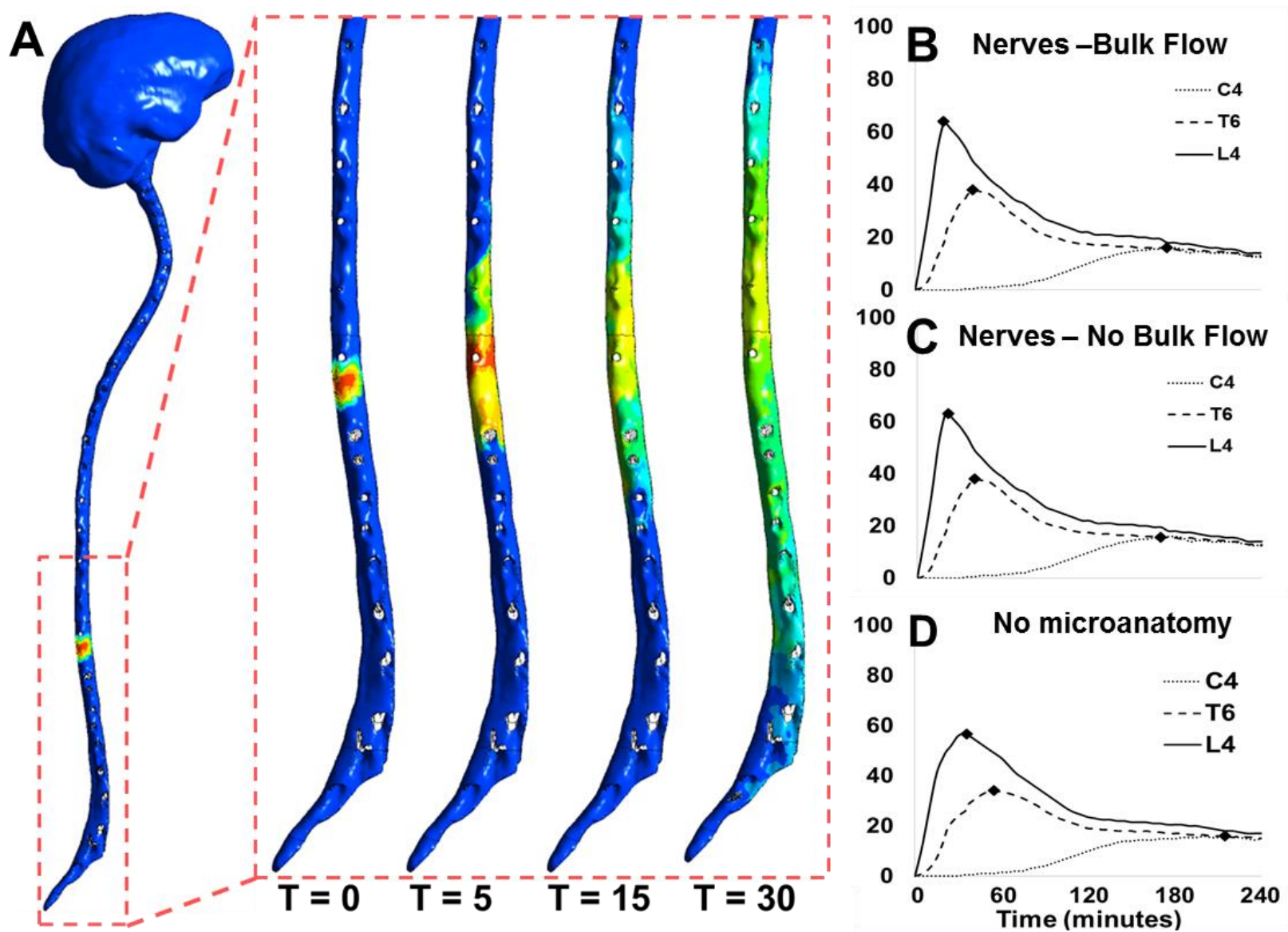
Table 1: Summary of Simulated and MR measured Flow Parameters

\begin{tabular}{|c|c|c|c|c|c|c|c|c|c|c|c|c|c|}
\hline & & \multirow{2}{*}{\begin{tabular}{|c|} 
Cross \\
section \\
A $\left(\mathrm{cm}^{2}\right)$ \\
\end{tabular}} & \multirow{2}{*}{$\begin{array}{c}\text { Diam. } \\
D_{\mathrm{h}}(\mathbf{c m})\end{array}$} & \multicolumn{2}{|c|}{$\begin{array}{c}\text { Peak Pressure } \\
\mathbf{P}_{\max }(\mathbf{P a})\end{array}$} & \multicolumn{2}{|c|}{$\begin{array}{l}\text { Peak velocity } \\
V_{\max }\left(\mathrm{cm} \mathrm{s}^{-1}\right)\end{array}$} & \multicolumn{2}{|c|}{$\begin{array}{c}\text { Peak vol. flow rate } \\
Q_{\max }\left(\mathrm{mL} \mathrm{s}^{-1}\right)\end{array}$} & \multirow{2}{*}{$\begin{array}{c}\text { Stroke } \\
\text { volume } \\
(\mathrm{mL})\end{array}$} & \multirow[t]{2}{*}{$\mathbf{R e}$} & \multirow[t]{2}{*}{ Wo } & \multirow{2}{*}{$\begin{array}{c}\text { Phase lag } \\
\phi \text { (deg) }\end{array}$} \\
\hline & & & & Systole & $\begin{array}{l}\text { Diastole } \\
\end{array}$ & Systole & Diastole & Systole & Diastole & & & & \\
\hline \multirow{7}{*}{ 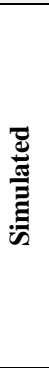 } & Cisterna & 4.21 & 1.83 & 223.08 & $\begin{array}{c}-90.99 \\
\end{array}$ & 0.98 & 0.51 & 4.13 & 2.17 & 0.763 & 179 & 22.84 & 7.2 \\
\hline & C4 & 1.98 & 0.91 & 200.77 & -81.89 & 1.23 & 0.64 & 2.45 & 1.26 & 0.443 & 112 & 11.42 & 10.8 \\
\hline & T1 & 3.49 & 1.26 & 167.31 & -68.24 & 0.51 & 0.26 & 1.77 & 0.91 & 0.322 & 64 & 15.78 & 25.2 \\
\hline & T6 & 1.27 & 0.80 & 129.39 & -51.64 & 1.07 & 0.56 & 1.36 & 0.71 & 0.248 & 86 & 10.54 & 32.4 \\
\hline & T11 & 0.84 & 0.66 & 82.54 & -33.67 & 1.49 & 0.77 & 1.25 & 0.64 & 0.228 & 98 & 8.29 & 43.2 \\
\hline & L4 & 1.50 & 0.96 & 44.62 & -18.2 & 0.69 & 0.34 & 1.04 & 0.50 & 0.202 & 67 & 12.07 & 61.2 \\
\hline & $\mathbf{S 3}$ & 2.08 & 1.08 & 17.85 & -7.28 & 0.15 & 0.07 & 0.35 & 0.17 & 0.067 & 16 & 13.47 & 64.8 \\
\hline \multirow{3}{*}{$\bar{\Sigma}$} & C4 & 1.92 & 0.89 & - & - & 1.14 & 0.73 & 2.19 & 1.43 & 0.439 & 102 & 11.19 & - \\
\hline & T6 & 1.44 & 0.83 & - & - & 0.83 & 0.60 & 1.17 & 0.83 & 0.244 & 69 & 10.36 & - \\
\hline & L4 & 2.19 & 1.04 & - & - & 0.47 & 0.29 & 1.04 & 0.68 & 0.199 & 49 & 13.04 & - \\
\hline
\end{tabular}

$\overline{R e}$ - Reynolds number at peak systole; Wo - Womersley number at peak systole; $\phi$ Phase lag between $P_{\max }$ and $V_{\max }$ at systole. 